\title{
The influence of type of vascular pedicle occlusion on the viability of skin island flaps. A postoperative quantitative assessment of flap survival in an experimental model in rats ${ }^{1}$
}

\author{
Rolf Gemperli', Alexandre Mendonça Munhoz ${ }^{\mathrm{II}}$
}

IAssociate Professor, Department of Surgery, Plastic Surgery Division, FMUSP, Sao Paulo, Brazil. Conception and design of the study, acquisition and interpretation of data.

IIFellow PhD degree, Postgraduate Program in Surgery, Department of Surgery, Plastic Surgery Division, FMUSP, Sao Paulo, Brazil. Interpretation of data, manuscript writing.

\section{ABSTRACT}

PURPOSE: To evaluate the role of pedicle occlusion on the viable area of a skin island flap.

METHODS: An epigastric skin island flap was performed in 160 Wistar rats. The animals were randomly divided into four groups: G-1: occlusion of inferior epigastric artery; G-2: inferior epigastric vein; G-3: inferior epigastric vessels (artery/vein). At varying times postoperatively $\left(2,3,4,5^{\text {th }}\right.$ day), animals from each group were randomly selected, and the pedicle was occluded. On the $10^{\text {th }}$. postop. day, the skin flaps were evaluated by templates to determine the percentage of surviving skin. The computer HPTM 9830A captured the photographs and the mean flap necrosis area was assessed.

RESULTS: An increasing area of flap survived with less area of necrosis with increased time of days intervals. In the G-1, the mean area of necrosis observed (two to five days) were 95.2, 60.8, 31 and $3.7 \%$ respectively. In the G-2 were 95.5, 57.6, 19.5 and 5.6\% respectively. In the G-3, a 100, 80, 32.4 and $14.9 \%$ of mean area was observed.

CONCLUSIONS: The arterial suppy/venous drainage is important for flap circulation up to five days following pedicle occlusion. After this period, the pedicle can be interrupted once the circulation through the flap margins has already been established.

Key words: Surgical Flaps. Graft Occlusion, Vascular. Skin. Rats. 


\section{Introduction}

Flap surgery has become a routine technique in modern plastic surgery and has allowed reconstruction of wounds defects around the world. In fact, the possibility to transfer skin and subcutaneous tissue has greatly improved the restoration of tissue defects following oncological resection, congenital malformations or major traumas ${ }^{1}$.

Basically, a flap is defined as an area of tissue that can be transferred and survives in its new location based on vascular pedicle. It is known that the initial survival of a skin island flap is determined by its vascular pedicle patency ${ }^{1,2}$. However, during the postoperative period there is the phenomenon of physiological neovascularization by means of neovessels derived from the surgical site and adjacent tissues. Thus, the critical point is the blood supply and the outcome can result in partial/total necrosis, and thereby resulting wound healing problems ${ }^{1,2}$. In skin flaps, an extended island exhibits an increasing gradient of ischemia and consequent regional tissue necrosis toward their distal margins ${ }^{3,4}$. In fact, this aspect may occur for a variety of reasons including insufficiency of blood supply secondary to mechanical obstruction of the pedicle (artery, vein or both), or ischemia-reperfusion injury, which occurs when blood flow is re-established to ischemic tissues ${ }^{4}$. Since complications related to flap transfer occur at a significant incidence, accurate assessment of flap status and the ability to determine whether or not it will survive is crucial ${ }^{1,2}$. Thus, understanding the events responsible for ischemia injuries helps us achieve better results in reconstructive surgeries, minimizing surgical morbidity.

Skin flap surgery has suggested different methods of investigating the importance of the axial artery/vein occlusion and ischemia ${ }^{3-10}$. Among the major studies evaluating flap neovascularization, there are few reports that analyze the type of pedicle occlusion and a postoperative quantitative assessment of skin flap survival. Thus, this study aims to clarify the possibility of survival of island skin flap in rats after occlusion of the pedicle arterial, venous, or both at predetermined postoperative times. The epigastric flap was chosen for this study because it has axial blood irrigation through the inferior surface epigastric vessels, easily accessible, and widely used in scientific research. In addition, assessing the time required for blood circulation of the flap becomes independent of the main vascular pedicle, favor the post surgical planning and greater predictability of results in island skin flaps.

\section{Methods}

The experimental protocol was approved by the Ethics Committee of the University of Sao Paulo, School of Medicine. All the procedures strictly followed the existing regulations about animal experimentation (Council for International Organization of Medical Sciences (CIOMS) ethical code for animal experimentation).

A total of 160 male Wistar rats, each weighing between 200 and 280 grams (mean 240 g), were housed at the FMUSP Experimental Surgery Laboratory Unit, where the surgical procedures were performed.

Surgical procedures began with anesthetizing the animals by ether inhalation, followed by trichotomy of the right inguinal and abdominal regions. Flap borders of 2.5 to $7.0 \mathrm{~cm}$ were marked on the abdominal skin from the median line (left lateral limit) and right inguinal region (lower limit). Analgesia during the surgery period was produced using intramuscular dipyrone $(30 \mathrm{mg} / \mathrm{kg})$. The surgical procedures were carried out under strictly aseptic and anti-septic conditions (Figure 1).
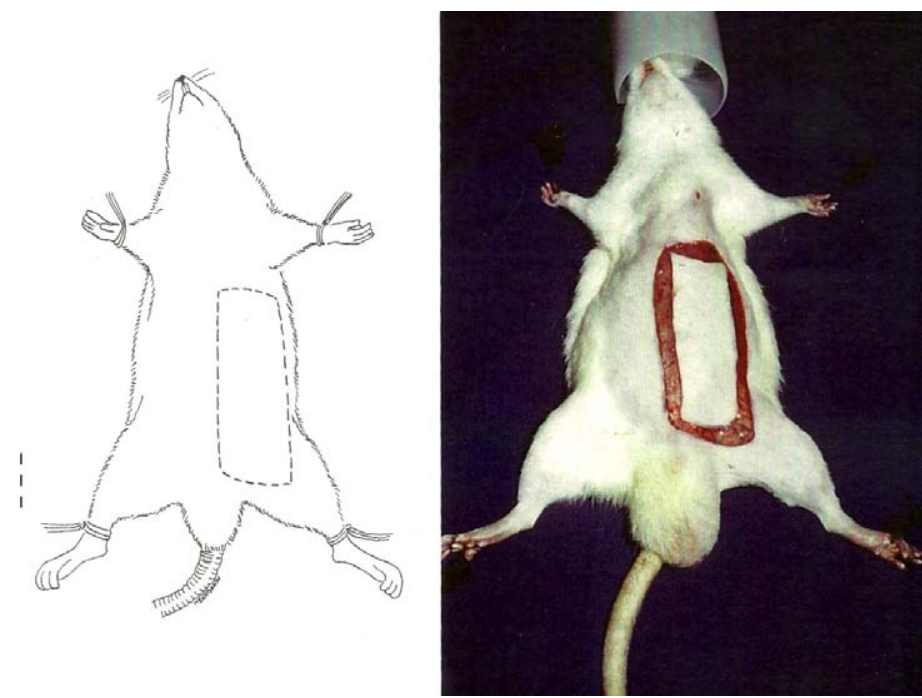

FIGURE 1 - Inferior epigastric abdominal flap borders of 2.5 to $7.0 \mathrm{~cm}$ were marked on the abdominal skin from the median line (left lateral limit) and right inguinal region (lower limit).

\section{Flap dissection}

The incision was made on the region from the borders marked on the skin to the aponeurotic plane, except for the inguinal region, where only the skin was incised, followed by dissection of the skin flap on the cranial-caudal direction, with clear sight of the vascular-nervous pedicle, making the procedure safer (Figures 2-3). 
The influence of type of vascular pedicle occlusion on the viability of skin island flaps. A postoperative quantitative assessment of flap survival in an experimental model in rats

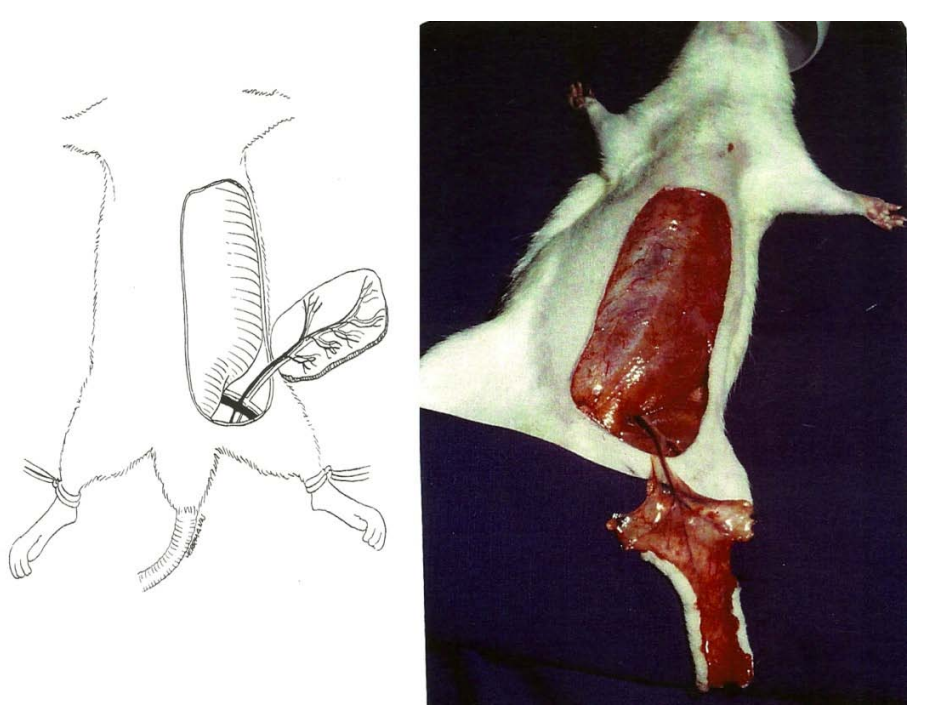

FIGURE 2 - The incision was made on the region from the borders marked on the skin to the aponeurotic plane.
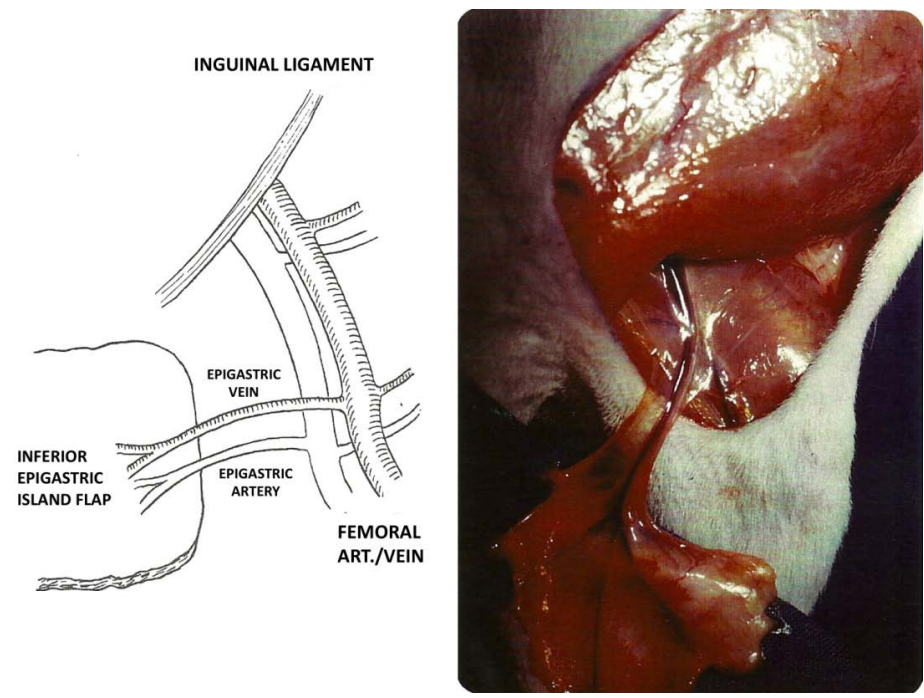

FIGURE 3 - The inguinal region: the vascular-nervous pedicle, inferior epigastric vessels.

A continuous suture was made using monofilament 5-0 nylon threads from the skin flap to its beds. Once the flap is denervated and has the possibility to auto cannibalize, a vest is fashioned from a strip of $\mathrm{x}$-ray film $(14 \mathrm{x} 4 \mathrm{~cm})$ in order to protect the abdominal flap area and allows trouble-free gavaging. A silicone sheet of $0.3 \mathrm{~mm}$ thickness and $7.0 \times 2.5 \mathrm{~cm}$ size was then interposed between the flap and the recipient bed in order to avoid vascularization of this region.

\section{Pedicle occlusion}

According to the pedicle vessel occluded, the animals were randomly divided into four groups:
GROUP 1: occlusion of inferior epigastric artery $(\mathrm{n}=40)$

GROUP 2: occlusion of inferior epigastric vein $(n=40)$

GROUP 3: occlusion of inferior epigastric vessels (artery and vein, $(\mathrm{n}=40))$

GROUP 4: control group $(n=40)$

At varying times postoperatively $\left(2,3,4\right.$ and $5^{\text {th }}$ postoperative day), animals from each group (1,2 and 3) were randomly selected, and once again anesthetized. At that time, the vascular-nervous bundle was dissected with the aid of a microsurgical microscope, and the artery, vein and the whole pedicle of this island flap was ligated and divided according to the group. The survival of the flap was thus dependent on the new channels, which had developed across the margins of the flap.

\section{Assessment of skin flap survival}

On the tenth postoperative day, the island skin flaps in all groups were evaluated. Viable skin was defined as a soft, pink, and warm to the touch flap area. Necrotic skin was invariably black and hardened seven days after injury; therefore, the distinction was unequivocal (Figure 4). The flap area was photographed and compared with that recorded on the first day of the experiment. Paper templates measured to the area of the flaps were prepared and utilized to determine the percentage of surviving skin. The images were analyzed by transposition of necrosis and total areas to template and comparison between areas. The computer HP ${ }^{\mathrm{TM}}$ 9830A captured the photographs and the mean flap necrosis area was then assessed for all groups (Figure 4 A-C). All the results are represented as mean, maximum and minimum values and standard deviation. The animals were sacrificed and under anaesthesia and after the collection of samples, the animals were put in a chamber and flushed with $\mathrm{CO}_{2}$ until cardiorespiratory arrest as per the guidelines of the American Veterinary Medical Association and the Federal Board of Veterinary Medicine.

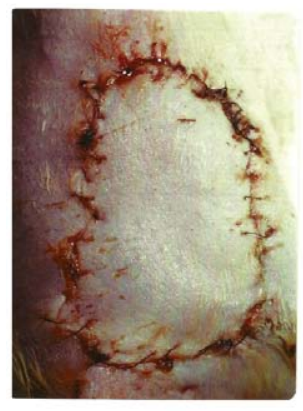

A

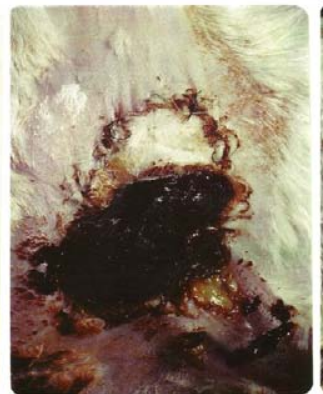

B

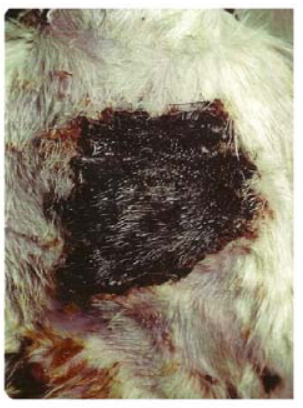

C
FIGURE 4 - Viable skin was defined as a soft, pink, and warm to the touch flap area (A). Necrotic skin was invariably black and hardened ten days after injury (B-C). 


\section{Statistical analysis}

The areas of flap necrosis were expressed as mean and standard deviation. The statistical analysis used in this study employed the technique of linear models. Multiple comparisons were made between percentage of necrosis and day of the pedicle ligature and percentage of necrosis and type of pedicle occlusion. The Duncan's Multiple Range Test analyzed the results observed in the 10th postoperative day for all sub-groups. The test were performed by statistical program software SAS (Statistical Analysis System).

\section{Results}

In the control group, the skin island flaps demonstrated complete survival with no area of necrosis. In the vessel occlusion groups (1, 2 and 3), an increasing area of each flap survived with less area of necrosis with increased time of postoperative days intervals (Figure 5).

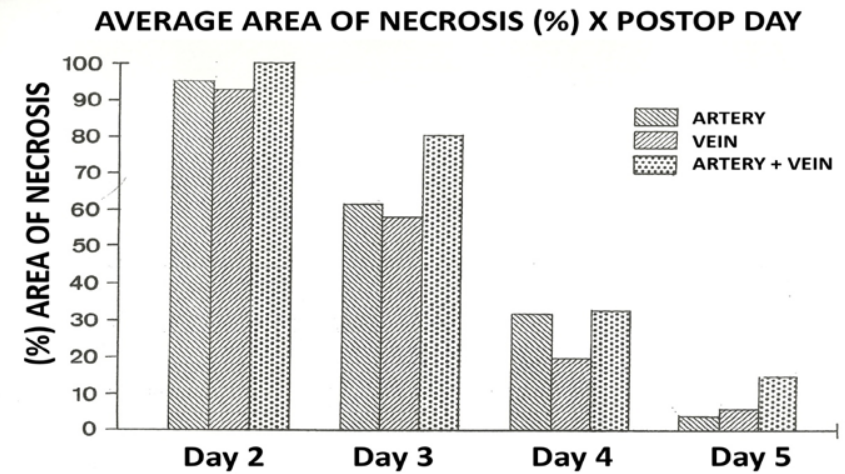

FIGURE 5 - In the control group, the skin island flaps demonstrated complete survival with no area of necrosis. In the vessel occlusion groups $(1,2$ and 3), an increasing area of each flap survived with less area of necrosis with increased time of postoperative days intervals.

In the Group I (epigastric artery occlusion), the flap showed marked cyanosis and congestion and different parts of the flap went on to partial necrosis. In this group, the mean area of necrotic skin flap observed after intervals of two to five days between flap elevation and vessel ligation were 95.2, 60.8, 31 and $3.7 \%$ respectively (Figure 6). In the Group II (epigastric vein occlusion), the mean area of necrotic skin flap observed following the same postoperative intervals were 95.5, 57.6, 19.5 and 5.6 $\%$ respectively (Figure 7). Similarly, in the group III (epigastric vessels, artery and vein occlusion), a 100, 80, 32.4 and $14.9 \%$ of mean area of necrotic skin flap was observed during the same period of follow-up (Figure 8). These results are summarized in Table 1.

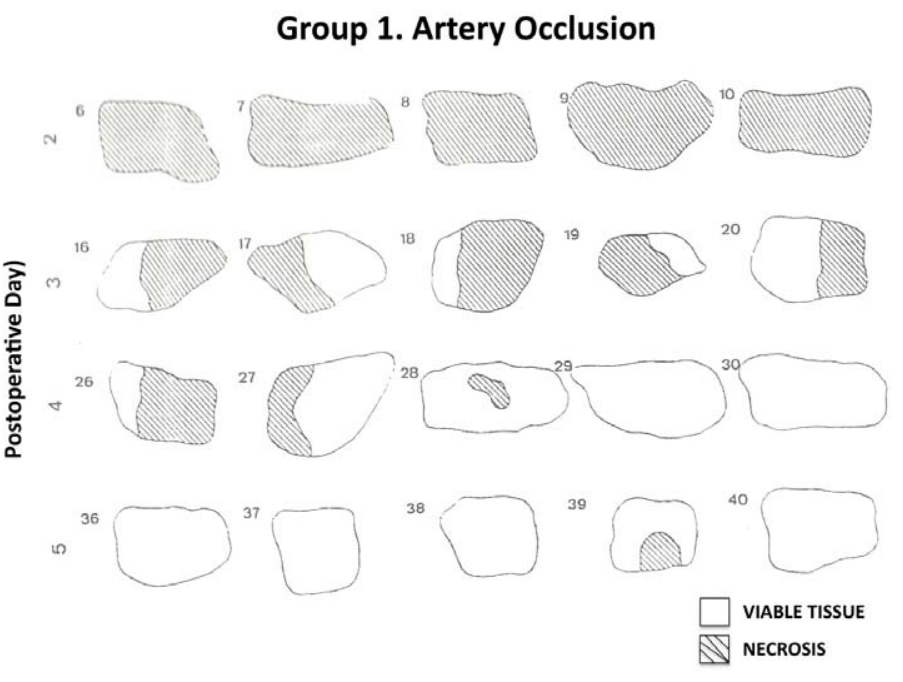

FIGURE 6 - Paper templates measured to the area of the flaps to determine the percentage of surviving skin. The images were analyzed by transposition of necrosis and total areas to template and comparison between areas. Group 1 (artery occlusion).

\section{Group 2. Vein Occlusion}

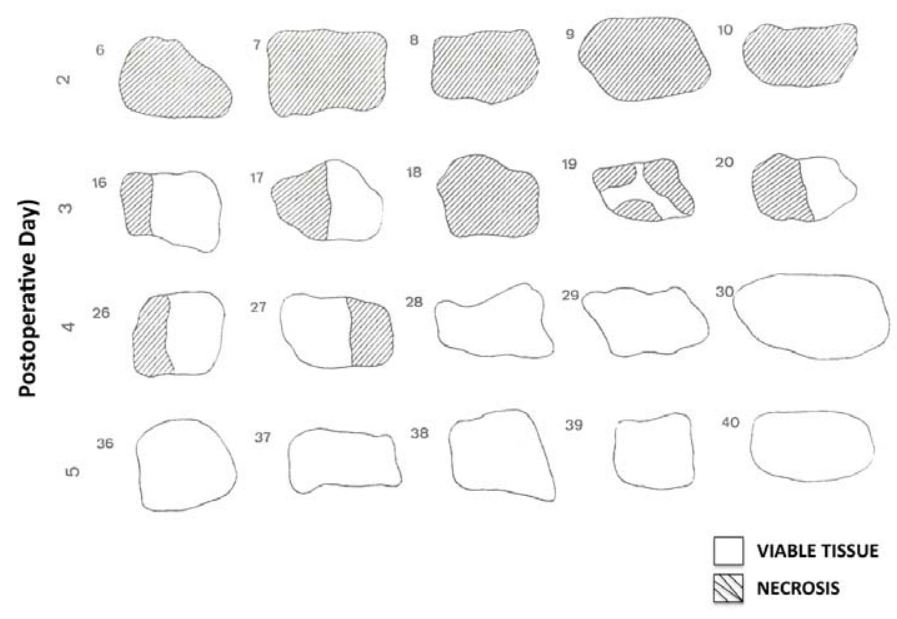

FIGURE 7 - Paper templates measured to the area of the flaps to determine the percentage of surviving skin. The images were analyzed by transposition of necrosis and total areas to template and comparison between areas. Group 2 (vein occlusion). 
The influence of type of vascular pedicle occlusion on the viability of skin island flaps. A postoperative quantitative assessment of flap survival in an experimental model in rats

\section{Group 2. Artery-Vein Occlusion}

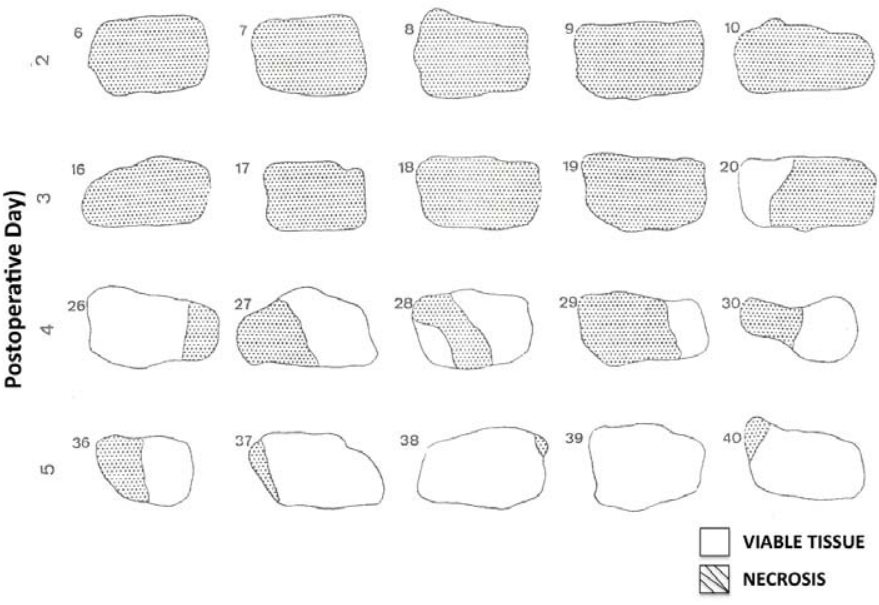

FIGURE 8 - Paper templates measured to the area of the flaps to determine the percentage of surviving skin. The images were analyzed by transposition of necrosis and total areas to template and comparison between areas. Group 3 (artery-vein occlusion).

TABLE 1 - Effect of pedicle oclusion on flap survival.

\begin{tabular}{rcrrrr}
\hline Group & Day & Mean (\%) & SD & Max Value & Min Value \\
\hline \multirow{4}{*}{ I } & 2 & 95.20 & 10.19 & 100.0 & 73.38 \\
& 3 & 60.82 & 22.02 & 100.0 & 37.53 \\
& 4 & 31.05 & 27.57 & 76.47 & 0.00 \\
& 5 & 3.72 & 7.85 & 19.46 & 0.00 \\
& & & & & \\
II & 3 & 92.57 & 12.64 & 100.0 & 66.70 \\
& 4 & 57.67 & 33.45 & 100.0 & 4.44 \\
& 5 & 19.50 & 17.59 & 39.46 & 0.00 \\
& & 5.61 & 11.82 & 28.70 & 0.00 \\
& 2 & & & & \\
III & 3 & 80.02 & 22.67 & 100.0 & 43.90 \\
& 4 & 32.48 & 18.97 & 74.77 & 8.01 \\
& 5 & 14.90 & 15.21 & 45.81 & 0.00 \\
\hline
\end{tabular}

Group 1: occlusion of inferior epigastric artery; Group 2: occlusion of inferior epigastric vein; Group 3: occlusion of inferior epigastric vessels (artery and vein) SD: Standard Deviation; Max Value: maximum value; Min Value: minimum value

Data were subjected to Duncan test for comparison of days (Table 2). The average percentages of flap necrosis significantly reduced progressively with increasing time of pedicle ligation. Using the Duncan test to compare the type of surgery and the percentage of necrotic area, it was observed that the percentage of necrosis occurred with ligation of the artery and vein were similar among themselves. However, the comparison with the ligation of group III (artery and vein) was different and with significantly higher incidence of necrosis (Table 3).

TABLE 2 - Mean percentages of flap necrosis and vascular ligation time (days).

\begin{tabular}{crrr}
\hline Day & Mean (\%) & SD & Grouping \\
\hline 2 & 95.92 & 9.57 & $\mathrm{~A}$ \\
3 & 66.17 & 27.53 & $\mathrm{~B}$ \\
4 & 27.67 & 21.88 & $\mathrm{C}$ \\
5 & 8.08 & 12.61 & $\mathrm{D}$ \\
\hline
\end{tabular}

SD: Standard Deviation

TABLE 3 - Mean percentages of flap necrosis and the type of vascular ligation (groups).

\begin{tabular}{crrr}
\hline Group & Mean (\%) & SD & Grouping \\
\hline I & 47.70 & 38.93 & A \\
II & 43.84 & 39.79 & A \\
III & 56.85 & 38.38 & B \\
\hline
\end{tabular}

Group 1: occlusion of inferior epigastric artery; Group 2: occlusion of inferior epigastric vein; Group 3: occlusion of inferior epigastric vessels (artery and vein) SD: Standard Deviation

\section{Discussion}

Experimental animal models have been used in surgical research to develop different surgical procedures, understanding of anatomy and physiology and refine surgical skills. The advantages of these models has been specially relevant in many plastic surgical techniques related to skin and musculocutaneous flaps that are studied in animals long before they are used in humans. Additionally, these skin flap models can also be used in experiments investigating rejection of allotransplanted composite tissue, which have importance in clinical situations. The behavior of experimental rat flaps may also give us relevant data that constrain the success of limb replantations, and as such, they form an important component of the scientific process in improving reconstructive surgery outcomes ${ }^{4}$.

In the present study, we utilized the experimental model of island skin flaps based on the inferior epigastric artery and vein as proposed by Strauch and Murray ${ }^{15}$ in 1967 and modified by Mckee et al. ${ }^{5}$ in 1981. In this flap model, usually the incision is made to the aponeurotic plane, except for the inguinal region, where only the skin is incised, followed by dissection of the skin flap, in order to preserve the vascular-nervous pedicle. Once the flap is denervated, we utilized a rat vest made from a strip of x-ray film in order to protect the abdominal flap area from autocannibalism as proposed 
by Pynn et $a l .{ }^{14}$. In addition, a silicone sheet is placed in between the abdominal wall and the flap to prevent neovascularization from the recipient bed.

The measurement of the surviving area of the skin flap was performed by the percentage of the original flap area. Viable skin was defined as a soft and pink flap area and necrotic skin was invariably black and hardened ten days after surgery. Previous studies used different methods to evaluate the necrosis area and the demarcation line between the health and non-viable tissue $^{8-11,16}$. In fact, Sasaki et al. ${ }^{8}$ advocated that the demarcation line was determined by midline measurement from base to necrotic border. Contrary, Patel et al. ${ }^{10}$ and Emerson et al. ${ }^{11}$, utilized the measurement from base to distal dye penetration line to define the survival length of the skin flap. Other studies, the skin survival was determined by measurement from base to most distal vascular filling using radiography ${ }^{16}$.

In our experience and similar as observed by other authors the pattern of skin necrosis rarely is represented by a line perpendicular to the long axis of the flap ${ }^{5,12}$. Thus, the necrosis evaluation can present some limitations once the area is not always exactly defined. In our sample on the tenth postoperative day the island skin flaps in all groups were evaluated. The flap area was photographed and compared with that recorded on the first day of the experiment. Paper templates measured to the area of the flaps were prepared and utilized to determine the percentage of surviving skin. The images were analyzed by transposition of necrosis and total areas to template and comparison between areas and the mean flap necrosis area was then assessed for all groups. According to this method, the area measurement was more easily definable and avoided ambiguity introduced by the possibility of equal areas of flap survival may be overlooked because of different particular measurement ${ }^{12}$.

Most of previous studies in flap survival following pedicle occlusion and neovascularization utilized the rabbit or rat models ${ }^{4-13}$. However, some variation and conflicting results exists in the survival observed in epigastric flaps in the rat mode ${ }^{5-7,13}$. In fact, Acland was the first investigator to correlate vessel ligation with flap survival ${ }^{5}$. The author demonstrated necrosis in free epigastric skin flaps in Sprague Dawley rats when both vessels were ligated at seven days, but complete survival when the vessels were occluded at three weeks. Nakajima used the experimental model in Wistar rats in order to evaluate the influence of the venous drainage following the ligation of the pedicle ${ }^{7}$. According to the author, after transfer of the flap, the venous drainage depend exclusively on the patency of the anastomosed vein until new vascular channels have developed and taken over the venous drainage of the skin flap.
Thus, the author advocates that it is crucial to know how long these new channels take to develop and hence the minimum patency period required of the anastomosed vein. Based on histological analysis the author observed that the revascularization began at the distal wound margin and spread toward the proximal margin. In addition, they observed vascular channels between the recipient bed and the island flap as early as three days after the procedure. By the $5^{\text {th }}$ postoperative day the whole wound margin was crowed with new vessels. Contrary, Serafin et al. ${ }^{9}$ in an experimental rabbit ear model, observed that five days were necessary for free flap survival when the vein was occluded. Besides the differences related to the experimental model, location and size of the flap that could partly explain the different result, the authors removed the perichondrium of the ear and electrocoagulated the cartilage in order to avoid revascularization from the recipient bed.

Mcgrath et $a l .{ }^{13}$, in an experimental model in Sprague Dawley rats utilized the fluorescein dye test to determine the earliest possible time a pedicle can be divided. The authors observed a complete survival of the skin flaps when the pedicle was occluded at 14 days. Similarly, Tsur et $a l .{ }^{6}$ utilized the same experimental model and observed those six days and earlier survival following ligation of only the artery or vein. McKee et al. ${ }^{5}$, observed in an experimental model in Wistar rats that both artery and vein ligation groups show equal survival from the second day and with complete survival after the third day. The authors concluded that survival of island flaps is not dependent on circulation through the vascular pedicle after three to five days.

Many factors such vessels anastomoses, state of the recipient bed or animal strain may be invoved in this differences. Our work substantiates that of McKee et al. ${ }^{5}$ and Tsur et al. ${ }^{6}$ and clearly defines the ability of an island flap to survive loss of one or both elements of its vascular pedicle with time. In our study, the results showed a high correlation in terms of flap survival and the postoperative day of the vessel occlusion. In the vessel occlusion groups an increasing area of each flap survived with less area of necrosis with increased time of postoperative days intervals. In the Group I, the mean area of necrotic skin flap observed after intervals of two to five days were 95.2, 60.8, 31 and $3.7 \%$ respectively. In the Group II, the mean area of necrotic skin flap observed following the same postoperative intervals were 95.5, $57.6,19.5$ and $5.6 \%$ respectively. Similarly, in the group III, a $100,80,32.4$ and $14.9 \%$ of mean area of necrotic skin flap was observed during the same period of follow-up. Thus, this present flap survival pattern indirectly support the concept of peripheral neovascularization and come from viable tissue around the island flap. Based on this results, one might surmise that recipient 
The influence of type of vascular pedicle occlusion on the viability of skin island flaps. A postoperative quantitative assessment of flap survival in an experimental model in rats

beds with a high vessel density (face) would vascularize a flap more quickly than ischemic areas (irradiated tissues). In spite of extrapolation from this experimental model to the clinical practice may not be suitable, consistent results can be seen within a model and were similar as observed by previous authors.

\section{Conclusions}

The neovascularization was sufficient to ensure flap survival and independence from the main pedicle (epigastric vessels). In addition, the development of new vessels was adequate to maintain almost total survival of the skin flaps by the end of five postoperative day of pedicle occlusion.

\section{References}

1. Kroll SS, Schusterman MA, Reece GP, Miller MJ, Evans GR, Robb GL, Baldwin BJ. Choice of flap and incidence of free flap success. Plast Reconstr Surg. 1996;98:459-3.

2. Kunselman AR, Landis JR, Yeramian P, Ingram D, Natarajan N, Benes CO, Wallemark C. A prospective study of microvascular freeflap surgery and outcome. Plast Reconstr Surg. 1998;102:711-1.

3. Yafi A, Vetter TS, Scholz T, Patel S, Saager RB, Cuccia DJ, Evans GR, Durkin AJ. Postoperative quantitative assessment of reconstructive tissue status in a cutaneous flap model using spatial frequency domain imaging. Plast Reconstr Surg. 2011;127(1):1171 .

4. Cubitt J, Pennington T, Wang C, Allen R, Bishop A, Sharland A. Reliable and reproducible murine models for commonly used abdominal plastic surgical flaps. J Reconstr Microsurg. 2012;28(3):161-6.

5. McKee NH, Clarke HM, Manktelow RT. Survival following vascular compromise in an island skin flap. Plast Reconstr Surg. 1981;67(2):200-4

6. Tsur H, Daniller A, Strauch B. Neovascularization of skin flaps: route and timing. Plast Reconstr Surg. 1980;66(1):85-1.

7. Nakajima T. How soon do venous drainage channels develop at the periphery of a free flap? A study in rats. Br J Plast Surg. 1978;31(4):300-8.

8. Sasaki A, Harii K. Lack of effect of isoxsuprine on experimental random flaps in the rat. Plast Reconstr Surg. 1980;66(1):105-8.

9. Serafin D, Shearin JC, Georgiade NG. The vascularization of free flaps: a clinical and experimental correlation. Plast Reconstr Surg. 1977;60(2):233-1.

10. Patel C, Marsili A, Sykes PJ. Augmentation of flap survival by thymoxamine. Br J Plast Surg. 1982;35(1):88-1.

11. Emerson DJ, Sykes PJ. The effect of prostacyclin on experimental random pattern flaps in the rat. Br J Plast Surg. 1981;34(3):264-6.

12. Nichter LS, Sobieski MW, Morgan RF, Rodeheaver G, Edlich RF. Quantitation of skin-flap survival: a computer-based method. Plast Reconstr Surg. 1984;73(4):684-6.

13. McGrath MH, Adelberg D, Finseth F. The intravenous fluorescein test: use in timing of groin flap division. J Hand Surg Am. 1979;4(1):19-2.

14. Pynn BR, McKee NH, Nigra CA, Howard CR. A protective rat vest. Plast Reconstr Surg. 1983;71(5):716-7.

15. Strauch B, Murray DE. Transfer of composite graft with immediate suture anastomosis of its vascular pedicle measuring less than $1 \mathrm{~mm}$. in external diameter using microsurgical techniques. Plast Reconstr Surg. 1967;40(4):325-9.

16. Hillelson RL, Glowacki J, Healey NA, Mulliken JB. A microangiographic study of hematoma-associated flap necrosis and salvage with isoxsuprine. Plast Reconstr Surg. 1980;66(4):528-3

\section{Correspondence:}

Alexandre Mendonça Munhoz, M.D.

Instituto de Ensino e Pesquisa - Hospital Sírio-Libanês

Rua Mato Grosso, 306/cj.1705-1706

01239-040 São Paulo - SP Brasil

munhozalex@uol.com.br

Received: March 20, 2013

Review: May 21, 2013

Accepted: June 18, 2013

Conflict of interest: none

Financial source: none

${ }^{1}$ Research performed at Experimental Surgery Laboratory Unit, Department of Surgery, School of Medicine, University of Sao Paulo (FMUSP), Brazil. Part of PhD degree thesis, Postgraduate Program in Surgery. Tutor: Prof. Rolf Gemperli. 